\title{
THE DERIVATIVE OF A SCHLICHT FUNCTION
}

\author{
A. J. LOHWATER, G. PIRANIAN and W. RUDIN
}

A. Bloch (see Nevanlinna $[4$, p. 138]) raised the question whether the derivative of a function which is meromorphic and of bounded characteristic in $|z|<1$ is also of bounded characteristic in $|z|<1$. Frostman $[1$, p. 181] answered this question by constructing a Blaschke product $B(z)$ whose derivative $B^{\prime}(z)$ has the property that for each $\theta$ the quantity $\lim _{r \rightarrow 1} B^{\prime}\left(r e^{i \theta}\right)$ fails to exist as a finite number. Since the radial limit of a function meromorphic and of bounded characteristic in $|z|<1$ exists and is finite, for almost all points on $|z|=1$ (see [4, p. 134]), Frostman's example answers Bloch's question in the negative. Recently, Rudin [5] has constructed a function $f(z)=\sum a_{n} z^{n}$, with $\Sigma\left|a_{n}\right|<\infty$, and with the property that $\lim _{r \rightarrow 1} f^{\prime}\left(r e^{i \theta}\right)=\infty$ for almost all $\theta$. In [6] he has exhibited another function $f(z)$, regular in $|z|<1$ and continuous in $|z| \leqq 1$, such that

$$
\int_{0}^{1}\left|f^{\prime}\left(r e^{i \theta}\right)\right| d r=\infty
$$

for almost all $\theta$. It should be noted that the function in [6] cannot possibly be schlicht, because if $f(z)$ is regular and schlicht in $|z|<1$, then

$$
\int_{0}^{1}\left|f^{\prime}\left(r e^{i \theta}\right)\right| d r<\infty
$$

for almost all $\theta$, by a theorem of Lavrentiev [2].

In the present note, we construct a schlicht function relevant to Bloch's question. The motivation for the construction comes from an example described by Lohwater and Piranian [3] in connection with a geometrical problem.

TheOREM. For a suitably increasing sequence $\left\{n_{p}\right\}$ of positive integers, the function

$$
f(z)=\int_{0}^{z} \exp \left\{\frac{1}{2} \sum_{p=1}^{\infty} w^{n_{p}}\right\} d w
$$

Received November 28, 1954. 
is regular in $|z|<1$, continuous and schlicht in $|z| \leqq 1$, and has for almost all $\theta$ the properties

$$
\begin{gathered}
\limsup _{r \rightarrow 1}\left|f^{\prime}\left(r e^{i \theta}\right)\right|=\infty, \\
\liminf _{r \rightarrow 1}\left|f^{\prime}\left(r e^{i \theta}\right)\right|=0, \\
\underset{r \rightarrow 1}{\lim \sup \arg f^{\prime}\left(r e^{i \theta}\right)}=+\infty, \\
\liminf _{r \rightarrow 1} \arg f^{\prime}\left(r e^{i \theta}\right)=-\infty .
\end{gathered}
$$

Moreover, the Taylor series of $f(z)$ converges absolutely on $|z|=1$.

Proof. We use the notation

$$
h_{p}(z)=\frac{1}{2} \sum_{k=1}^{p} z^{n_{k}}, \quad g_{p}(z)=\exp h_{p}(z), \quad f_{p}(z)=\int_{0}^{z} g_{p}(w) d w,
$$

and observe that

$$
f_{p+1}(z)-f_{p}(z)=\int_{0}^{z} g_{p}(w)\left[\exp \left\{\frac{1}{2} w^{n_{p+1}}\right\}-1\right] d w .
$$

We choose $n_{1}=1$ and define the further elements of the sequence $\left\{n_{p}\right\}$ as follows: having chosen $n_{1}, \ldots, n_{p}$, we write

$$
a_{p}=2 /\left(n_{1}+n_{2}+\ldots+n_{p}\right)
$$

and choose $n_{p+1}$ so that $n_{p+1} / n_{p}$ is an odd integer, and large enough so that

$$
\left|f_{p+1}(z)-f_{p}(z)\right|<a_{p} e^{-p-3} \quad(|z| \leqq 1) ;
$$

by (6), this construction is always possible. It follows from (8) that the sequence $\left\{f_{p}(z)\right.$ ) converges uniformly in $|z| \leqq 1$ to the function $f(z)$ defined by (1), and hence that $f(z)$ is regular in $|z|<1$ and continuous in $|z| \leqq 1$.

To show that $f(z)$ is schlicht in $|z| \leqq 1$, let $z_{1} \neq z_{2},\left|z_{1}\right| \leqq 1,\left|z_{2}\right| \leqq 1$; and let $p$ be that integer for which

By (8),

$$
a_{p}<\left|z_{1}-z_{2}\right| \leqq a_{p-1} .
$$

for $|z| \leqq 1$, whence

$$
\left|f(z)-f_{p}(z)\right|<\sum_{k=p}^{\infty} a_{k} e^{-k-3}<a_{p} e^{-p-2}
$$

$$
\left|f\left(z_{1}\right)-f\left(z_{2}\right)\right|>\left|f_{p}\left(z_{1}\right)-f_{p}\left(z_{2}\right)\right|-2 a_{p} e^{-p-2} .
$$


Also,

$$
f_{p}\left(z_{1}\right)-f_{p}\left(z_{2}\right)=\int_{z_{2}}^{z_{1}} g_{p}(w) d w=\int_{z_{2}}^{z_{1}} \exp \left\{h_{p-1}(w)+\frac{1}{2} w^{n p}\right\} d w,
$$

where the path of integration can be taken as the rectilinear segment from $z_{1}$ to $z_{2}$. Since

$$
\left|h_{p-1}{ }^{\prime}(z)\right| \leqq\left(n_{1}+n_{2}+\ldots+n_{p-1}\right) / 2 \quad(|z| \leqq 1)
$$

and $\left|z_{1}-z_{2}\right| \leqq a_{p-1}$, it follows from (7) that, as $w$ passes from $z_{1}$ to $z_{2}$, the argument of the integrand in (10) varies over an interval whose length is less than $1+1<\frac{2}{3} \pi$. Consequently the modulus of the integral in (10) exceeds

$$
\cos \frac{1}{3} \pi \cdot\left|z_{1}-z_{2}\right| \cdot \min \left|g_{p}(z)\right|>\frac{1}{2} a_{p} e^{-p}
$$

Applying this estimate to (9), we get

$$
\left|f\left(z_{1}\right)-f\left(z_{2}\right)\right|>\frac{1}{2} a_{p} e^{-p}-2 a_{p} e^{-p-2}=\frac{1}{2} a_{p} e^{-p-2}\left(e^{2}-4\right)>0,
$$

and therefore $f(z)$ is schlicht in $|z| \leqq 1$.

Next, we observe that (1) implies the relations

$$
\begin{aligned}
& \log \left|f^{\prime}\left(r e^{i \theta}\right)\right|=\frac{1}{2} \sum_{k=1}^{\infty} r^{n_{k}} \cos n_{k} \theta, \\
& \arg f^{\prime}\left(r e^{i \theta}\right)=\frac{1}{2} \sum_{k=1}^{\infty} r^{n_{k}} \sin n_{k} \theta .
\end{aligned}
$$

We denote by $E_{2}$ and $E_{3}$, respectively, the sets on $[0,2 \pi]$ on which (2) and (3) hold. Since the coefficients of the lacunary series $\Sigma \cos n_{k} \theta$ do not tend to zero, the Abel transform of the series is an unbounded function, for almost all $\theta$, so that $m\left(E_{2} \cup E_{3}\right)=2 \pi$ (for the details of this see, for example, [7, pp. 119-122]; and note that there the hypothesis of the convergence of the transform is not used, except inasmuch as it implies the boundedness of the transform).

Since $\left\{n_{k}\right\}$ is a sequence of odd integers, $\theta$ belongs to $E_{2}$ if and only if $\theta+\pi$ belongs to $E_{3}$; hence $m\left(E_{2}\right)=m\left(E_{3}\right)$. Since $n_{k+1} / n_{k}$ is an integer, both $E_{2}$ and $E_{3}$ are periodic with arbitrarily small periods $2 \pi / n_{k}$; consequently $m\left(E_{i}\right)(i=2,3)$ can have only one of the values 0 and $2 \pi$. Thus we conclude that $m\left(E_{2}\right)=m\left(E_{3}\right)=2 \pi$, and (2) and (3) hold for almost all $\theta$. An analogous discussion of $\Sigma \sin n_{k} \theta$ establishes (4) and (5).

Finally, we note that $f(z)=\sum a_{n} z^{n}$, where $a_{n} \geqq 0$. Since $\lim _{r \rightarrow 1} f(r)$ is finite, $\Sigma a_{n}<\infty$, and the proof is complete. 


\section{REFERENCES}

1. O. Frostman, Sur les produits de Blaschke, Kungl. Fysiografiska Sällskapets i Lund Förhandlingar [Proc. Roy. Physiog. Soc. Lund] 12 (1942) no. 15, 169-182 (=Meddelanden frản Lunds Universitets Matematiska Seminarium 5 (1943), 1-14).

2. M. Lavrentiev, Sur la correspondance entre les frontières dans la représentation conforme, Mat. Sbornik 36 (1929), 112-115.

3. A. J. Lohwater and G. Piranian, Linear accessibility of boundary points of a Jordan region, Comment. Math. Helv. 25 (1951), 173-180.

4. R. Nevanlinna, Le théorème de Picard-Borel et la théorie des fonctions méromorphes, Paris, 1929.

5. W. Rudin, On a problem of Bloch and Nevanlinna, to appear in Proc. Amer. Math. Soc.

6. W. Rudin, On the radial variation of analytic functions, to appear in Duke Math. J.

7. A. Zygmund, Trigonometrical series, Warszawa, 1935.

UNIVERSITY OF MICHIGAN, ANN ARBOR, MICH., U.S.A., AND

UNIVERSITY OF ROCHESTER, ROCHESTER, N.Y., U.S.A. 